\title{
Comparison Between Calcaneus Quantitative Ultrasound and the Gold Standard DXA In the Ability to Detect Osteoporosis in COPD Patients
}

\section{Wandee Chanprasertpinyo}

School of Medicine, Walailak University

\section{Chuchard Punsawad}

School of Medicine, Walailak University

Naparat Sukkriang

School of Medicine, Walailak University

Rapheeporn Khwanchuea

School of Medicine, Walailak University

\section{Pirada Yincharoen}

School of Medicine, Walailak University

Chaiwat Rerkswattavorn ( $\nabla$ chaiwat.re@mail.wu.ac.th )

School of Medicine, Walailak University

\section{Research Article}

Keywords: Chronic obstructive pulmonary disease, Osteoporosis, Quantitative ultrasound

Posted Date: February 2nd, 2021

DOI: https://doi.org/10.21203/rs.3.rs-153388/v1

License: (c) (1) This work is licensed under a Creative Commons Attribution 4.0 International License. Read Full License 


\section{Abstract}

Introduction

Osteoporosis is a prevalent comorbidity in patients with COPD that is usually underrecognized and hence undertreated. Compared to the gold standard dual-energy X-ray absorptiometry (DXA), calcaneus quantitative ultrasound (QUS) is less expensive, more portable, and more accessible, especially in less developed countries. The aim of this study was to investigate the ability of calcaneus QUS to detect osteoporosis in patients with COPD.

\section{Methods}

This cross-sectional study enrolled 67 males older than 50 years with clinically stable COPD. DXA scans of the lumbar spine (L2-4) and femoral neck were performed. QUS of the right calcaneus (AOS-100) were used to assess the broadband ultrasound attenuation (BUA), speed of sound (SOS), osteo sonoassessment index (OSI), and T-score. When the T-score was $\leq-2.5$, osteoporosis was diagnosed by both DXA and QUS.

Results

Forty-eight patients $(71.6 \%)$ had DXA T-scores $\leq-2.5$ at either the lumbar spine or femoral neck. All QUS parameters (BUA, SOS, OSI, and T-score) could discriminate DXA-determined osteoporosis (the area under the curve varied from 0.64 to 0.83 ). The QUS T-score was significantly moderately correlated with the DXA T-score at both the femoral neck $(r=0.62)$ and lumbar spine $(r=0.53)$. The sensitivity and specificity of QUS in identifying osteoporosis were $10.4 \%$ and $94.7 \%$, respectively. The positive and negative predictive values were $83.3 \%$ and $29.5 \%$, respectively.

Conclusion

The calcaneus QUS T-score had the ability to identify osteoporosis with an acceptable level of accuracy. However, the sensitivity and specificity of the QUS T-score were not sufficiently high to serve as an alternative diagnostic tool to DXA.

\section{Introduction}

Chronic obstructive pulmonary disease (COPD) is a significant public health problem resulting in socioeconomic burden due to its high prevalence, morbidity, and mortality ${ }^{1,2}$. Osteoporosis is a significant morbidity in COPD patients that is usually underrecognized and hence undertreated ${ }^{3}$. Patients are typically asymptomatic until fractures occur. Osteoporosis and osteoporosis fractures are extremely common in COPD patients. The overall prevalence of osteoporosis in COPD patients, according to previous studies, ranges from $14-66 \%$. The significant associated factors include being underweight (body mass index $(\mathrm{BMI})<18.5 \mathrm{~kg} / \mathrm{m}^{2}$ ), the presence of sarcopenia, an older age, a high Global Initiative 
for Chronic Obstructive Lung Disease (GOLD) stage, a low physical activity level, vitamin D deficiency, a low fat-free mass index (FFMI) and the use of systemic steroids ${ }^{4,5}$.

Vertebral compression fractures (VCFs) are the most common type of osteoporosis-induced fractures and are common in COPD patients, with a prevalence ranging from $24-79 \%{ }^{6}$. VCFs can cause kyphosis and subsequently result in impaired lung function in COPD patients. Moreover, osteoporosis fractures are associated with pain and lead to decreased mobility, which thereby impairs individuals' ability to perform activities of daily living (ADL) ${ }^{7}$. Therefore, the early detection of osteoporosis is considerably essential for the management of patients with COPD.

The gold standard for the diagnosis of osteoporosis is the measurement of bone mass density (BMD) using dual-energy X-ray absorptiometry (DXA). However, the accessibility of DXA is limited due to the increasing demand for bone health assessments with population aging. Thus, there is a long waiting time, which can delay diagnosis and treatment in patients with osteoporosis. Moreover, DXA is relatively costly and involves a low level of ionizing radiation.

Quantitative ultrasound (QUS) is an attractive method for bone health assessments and has gained much attention in recent years. It is portable and inexpensive, does not emit ionizing radiation, and is more accessible to the public, particularly in less developed countries ${ }^{8}$. The calcaneus is the only site recommended by the International Society of Clinical Densitometry (ISCD) for QUS ${ }^{9}$. A recent metaanalysis demonstrated that calcaneus QUS can potentially be used as a prescreening tool for the assessment of osteoporosis ${ }^{10}$. The role of QUS in screening for osteoporosis in COPD patients has not been studied.

Therefore, the aims of this study were to investigate the ability of calcaneus QUS to identify COPD patients with DXA-determined osteoporosis.

\section{Methods}

\section{Study design and population}

This is a cross-sectional study that was conducted from March 2019 to April 2020. The study was conducted in accordance with the Declaration of Helsinki and the Human Research Ethics Committee of Walailak University approved the protocol (WUEC-19-059-01). Informed consent was obtained from all enrolled patients before any study-related procedures were performed. We recruited patients with a known diagnosis of COPD from the outpatient COPD clinic at Thasala Hospital, a primary care hospital located in the rural area of Nakhon Si Thammarat, Thailand. Patients were considered eligible for this study if they met the following inclusion criteria: were male, were older than 50 years old, and had clinically stable COPD confirmed by postbronchodilator FEV $1 / F V C<0.7$, where clinically stable was defined as the absence of exacerbation in the previous four weeks. Patients were excluded if they had one or more of the following criteria: were diagnosed with osteoporosis or taking anti-osteoporosis drugs, including 
calcium or vitamin D supplements; had asthma, a rheumatic disease, chronic liver or renal disease, primary or secondary hyperparathyroidism, thyroid dysfunction, or Cushing syndrome; or had taken oral corticosteroids in the preceding six weeks.

\section{Measurements}

Demographic data were collected by interviewing the patients and reviewing their medical records. An internist from the researcher team interviewed the patients and collected the following data: smoking history, symptom assessment using the COPD Assessment Test (CAT), and physical activity level using the Global Physical Activity Questionnaire (GPAQ). The patients' medical records were reviewed to determine their current and previous medications, spirometry values, history of hospitalization due to COPD exacerbation in the past 12 months, and comorbidities. The comorbidities were summarized by the Charlson comorbidity index ${ }^{11}$.

BMD was measured by two methods at the same visit for all patients. First, DXA scans (Osteosys Dexxum T, OsteoSys, Korea) of the second to the fourth lumbar vertebrae (L2-4) and the right femoral neck were taken. Second, a QUS of the right calcaneus was performed using the Acoustic Osteo-Screener ultrasound device (AOS-100, Aloka Co., Ltd., Japan). QUS was used to assess the following parameters: broadband ultrasound attenuation (BUA), speed of sound (SOS), osteo sono-assessment index (OSI), and T-score. The QUS T-score of the investigated device is determined by comparing the OSI with the mean OSI of a reference population. The OSI has been shown to be correlated with the BMD measured by DXA 12.

According to the WHO, osteoporosis is present when BMD is 2.5 standard deviations (SDs) or more below the average value for normal young adults (a T-score of $\leq-2.5 \mathrm{SD}$ ). Osteopenia was recorded when the T-score was between - 1.0 SD and - 2.5 SD. T-scores higher than - 1.0 SD were considered normal (a Tscore $\geq 1)$. Patients with an abnormal DXA T-score were referred to an orthopedist for appropriate treatment. In this study, we used the DXA T-score criteria to interpret the QUS T-score.

We measured BMI and fat-free mass with bioelectrical impedance analysis (TANITA SC-330, Tanita Corp., Japan). The FFMI was calculated as the FFM divided by the square of the patient's height. BMI was categorized as low $\left(<18.5 \mathrm{~kg} / \mathrm{m}^{2}\right)$, normal $\left(18.5-24.9 \mathrm{~kg} / \mathrm{m}^{2}\right)$, overweight $\left(25.0-29.9 \mathrm{~kg} / \mathrm{m}^{2}\right)$, or obese $(>$ $\left.30 \mathrm{~kg} / \mathrm{m}^{2}\right)^{13}$. FFMI was considered to be depleted when FFMI $<16 \mathrm{~kg} / \mathrm{m}^{2}$ in the men ${ }^{14}$. A blood sample was drawn and stored at $-80^{\circ} \mathrm{C}$ in a freezer for subsequent biochemical analysis. Serum 25hydroxyvitamin D [25(OH)D] was measured by an enzyme-linked immunosorbent assay (ELISA) kit (ab213966 25-OH Vitamin D ELISA kit, Abcam, UK).

\section{Statistical analysis}

Statistical Package for Social Sciences (SPSS), version 26, and STATA, version 14.1, were used for data analyses. The descriptive statistics are presented as frequencies with percentages and means \pm SDs or medians with interquartile ranges (IQRs). Independent T-tests and chi-square tests were used to compare the continuous and categorical variables, respectively. Logistic regression was performed to identify the 
significant factors associated with osteoporosis. Pearson's correlation analysis was performed to assess the correlation between the DXA T-score and calcaneus QUS T-score. Receiver operating characteristic (ROC) analysis was performed, and the areas under the curves (AUCs) were calculated to compare the ability of calcaneus QUS with that of DXA to detect osteoporosis.

\section{Results}

\section{Patient characteristics}

Among the 89 patients who met all the inclusion and exclusion criteria, 67 patients responded to participate in this study. A comparison of the demographic characteristics of the osteoporotic and nonosteoporotic groups is summarized in Table 1. The mean age of the whole cohort was 69.6 years, and $52 \%$ of the patients were older than 70 years. The participants in the osteoporotic group were slightly older than those in the nonosteoporotic group, but the difference was not significant. Almost all patients (94\%) had used inhaled corticosteroids in the past year. The duration and dose of inhaled corticosteroids did not significantly differ between the two groups. According to the GOLD classification system of airflow limitation severity, most of the patients in the two groups had stage II limitations. The median CAT score, number of exacerbations in the previous year, and number of oral corticosteroid users did not differ between the two groups. The mean BMI of all the participants was $21.27 \pm 3.8 \mathrm{~kg} / \mathrm{m}^{2}$, and the majority of the patients $\left(73.1 \%\right.$ ) had a BMI $\geq 18.5 \mathrm{~kg} / \mathrm{m}^{2}$. The mean FFMl of all participants was $17.35 \pm 1.92 \mathrm{~kg} / \mathrm{m}^{2}$, and 18 patients (26.9\%) had a depleted FFMI (FFMI $\left.<16 \mathrm{~kg} / \mathrm{m}^{2}\right)$. The mean BMI and FFMI values were significantly lower in the osteoporotic group than in the nonosteoporotic group. All calcaneus parameters other than BUA were significantly lower in the osteoporotic group. In both groups, the femoral T-score was lower than the lumbar spine T-score.

\section{Table 1 Baseline characteristics of the study population}




\begin{tabular}{|c|c|c|c|}
\hline & $\begin{array}{l}\text { Osteoporosis } \\
\mathrm{N}=48\end{array}$ & $\begin{array}{l}\text { Nonosteoporosis } \\
\mathrm{N}=19\end{array}$ & $\mathrm{p}$-value \\
\hline Age, years & $70.21 \pm 8.97$ & $68 \pm 7.62$ & 0.348 \\
\hline Body mass index $\left(\mathrm{kg} / \mathrm{m}^{2}\right)$ & $20.35 \pm 3.62$ & $23.62 \pm 3.26$ & $0.001 *$ \\
\hline \multicolumn{4}{|l|}{ Tobacco use } \\
\hline Current smoker, no (\%) & $8(16.7 \%)$ & $5(26.3 \%)$ & \multirow[t]{3}{*}{0.496} \\
\hline Ex-smoker, no (\%) & $39(81.3 \%)$ & $13(68.4 \%)$ & \\
\hline Pack-years & $\begin{array}{l}28.50(10 \\
60)\end{array}$ & $30(7.5,40)$ & \\
\hline Charlson comorbidity index & $3.73 \pm 1.14$ & $3.53 \pm 1.12$ & 0.513 \\
\hline Duration of inhaled corticosteroid, year & $\begin{array}{l}4.42(1.25 \\
7.63)\end{array}$ & $2.75(1,7)$ & 0.587 \\
\hline Fluticasone equivalent dose, mcg/day & & $250(250,500)$ & 0.744 \\
\hline Oral corticosteroid uses in the past year, no (\%) & $\begin{array}{l}500(250 \\
500)\end{array}$ & $6(31.6 \%)$ & 0.766 \\
\hline GOLD COPD staging, no (\%) & $17(35.4 \%)$ & & \\
\hline I: $\mathrm{FEV}_{1} \geq 80 \%$ predicted & & $4(21.1 \%)$ & 0.289 \\
\hline II: FEV $150-80 \%$ predicted & $7(14.6 \%)$ & $10(52.6 \%)$ & \\
\hline III: FEV ${ }_{1} 30-50 \%$ predicted & $21(43.8 \%)$ & $\begin{array}{l}5(26.3 \%) \\
0(0 \%)\end{array}$ & \\
\hline IV: $\mathrm{FEV}_{1}<80 \%$ predicted & $12(25 \%)$ & & \\
\hline & $8(16.7 \%)$ & $11(1,16)$ & 0.411 \\
\hline COPD Assessment Test (CAT $\left.{ }^{\mathrm{TM}}\right)$ score & $12(4,18)$ & $0(0,1)$ & 0.514 \\
\hline Numbers of exacerbation in the previous year & $0(0,1)$ & $18.38 \pm 1.42$ & $0.002 *$ \\
\hline
\end{tabular}


FFMI $\left(\mathrm{kg} / \mathrm{m}^{2}\right)$

$16.94 \pm 1.95 \quad 840(280,2520) \quad 0.850$

Global Physical Activity Questionnaire total activity,

metabolic equivalent intensity $\mathrm{min} /$ week

$840(380$,

1920)

Serum 25(OH)D level, ng/ml

$7.86 \pm 0.28$

0.531

Calcaneus QUS

sos, $\mathrm{m} / \mathrm{s}$

$7.80 \pm 0.37$

BUA, dB/MHz

$1553.47 \pm 25.79$

$0.004^{*}$

$60.76 \pm 18.22$

0.140

OSI

T-score

$1533.15 \pm$

$2.76 \pm 0.67$

0.040 *

17.97

$-0.48 \pm 1.55$

0.012 *

$53.38 \pm$

Femoral neck T-score

18.18

$2.41 \pm 0.30$

$-1.37 \pm 1.06$

$<0.001^{*}$

Lumbar spine T-score

$-1.51 \pm 0.88$

$-0.63 \pm 1.50$

$<0.001$ *

$-3.49 \pm 1.04$

$-3.21 \pm 1.33$

Values are mean $\pm S D$ or median (range). Abbreviations: FEV1 = force expiratory volume in the first second, $\mathrm{BMI}=$ body mass index, $\mathrm{FFMI}$ = fat-free mass index, SABA = short-acting beta-agonists, SAMA =short acting muscarinic antagonists, LAMA = long-acting muscarinic antagonist, $25(\mathrm{OH}) \mathrm{D}=25$ hydroxyvitamin $\mathrm{D}$, * $\mathrm{p}$-value is significant.

\section{Prevalence of osteoporosis determined by DXA and factors associated with osteoporosis}

The mean T-score and prevalence of osteoporosis and osteopenia are presented in Table 2. The mean Tscore of the femoral neck $(-2.89 \pm 1.42)$ was significantly lower than that of the lumbar spine $(-2.48 \pm 1.8$, $p=0.002)$. The prevalence of osteoporosis and that of osteopenia at the femoral neck were $64.2 \%$ and $28.4 \%$, respectively. The prevalence of osteoporosis and that of osteopenia at the lumbar spine were $50.7 \%$ and $34.3 \%$, respectively. When the lowest T-score at either the lumbar spine or femoral neck was used for diagnosis, there were 48 patients (71.6\%) with osteoporosis and 16 patients $(23.9 \%)$ with osteopenia. 
Table 2: Bone mineral density and prevalence of osteoporosis and osteopenia

\begin{tabular}{|lllll|}
\hline Measurement & Mean T-score (SD) & $\begin{array}{l}\text { Osteoporosis } \\
\mathrm{n}(\%)\end{array}$ & $\begin{array}{l}\text { Osteopenia } \\
\mathrm{n}(\%)\end{array}$ & $\begin{array}{l}\text { Normal } \\
\mathrm{n}(\%)\end{array}$ \\
\hline DXA & & & & \\
Femoral neck & $-2.89(1.42)$ & $43(64.2)$ & $19(28.4)$ & $5(7.5)$ \\
Lumbar spine & $-2.48(1.80)$ & $34(50.7)$ & $23(34.3)$ & $10(14.9)$ \\
Calcaneus QUS & $-1.22(1.19)$ & $6(9.0)$ & $39(58.2)$ & $22(32.8)$ \\
\hline
\end{tabular}

Osteopenia: T-score between -1.0 SD and -2.5 SD, Osteoporosis: T-score <-2.5 SD

The significant risk factors associated with osteoporosis were BMI and FFMI, as shown in Table 3. The COPD patients with a low BMI (BMI $\left.<18.5 \mathrm{~kg} / \mathrm{m}^{2}\right)$ had an almost 9-fold increased risk of osteoporosis than did the patients with a normal BMI. The patients who had an FFMl $<17 \mathrm{~kg} / \mathrm{m}^{2}$ had a five times higher risk of osteoporosis than did those with an FFMI greater than $17 \mathrm{~kg} / \mathrm{m}^{2}$.

\section{Table 3: Factors associated with osteoporosis}




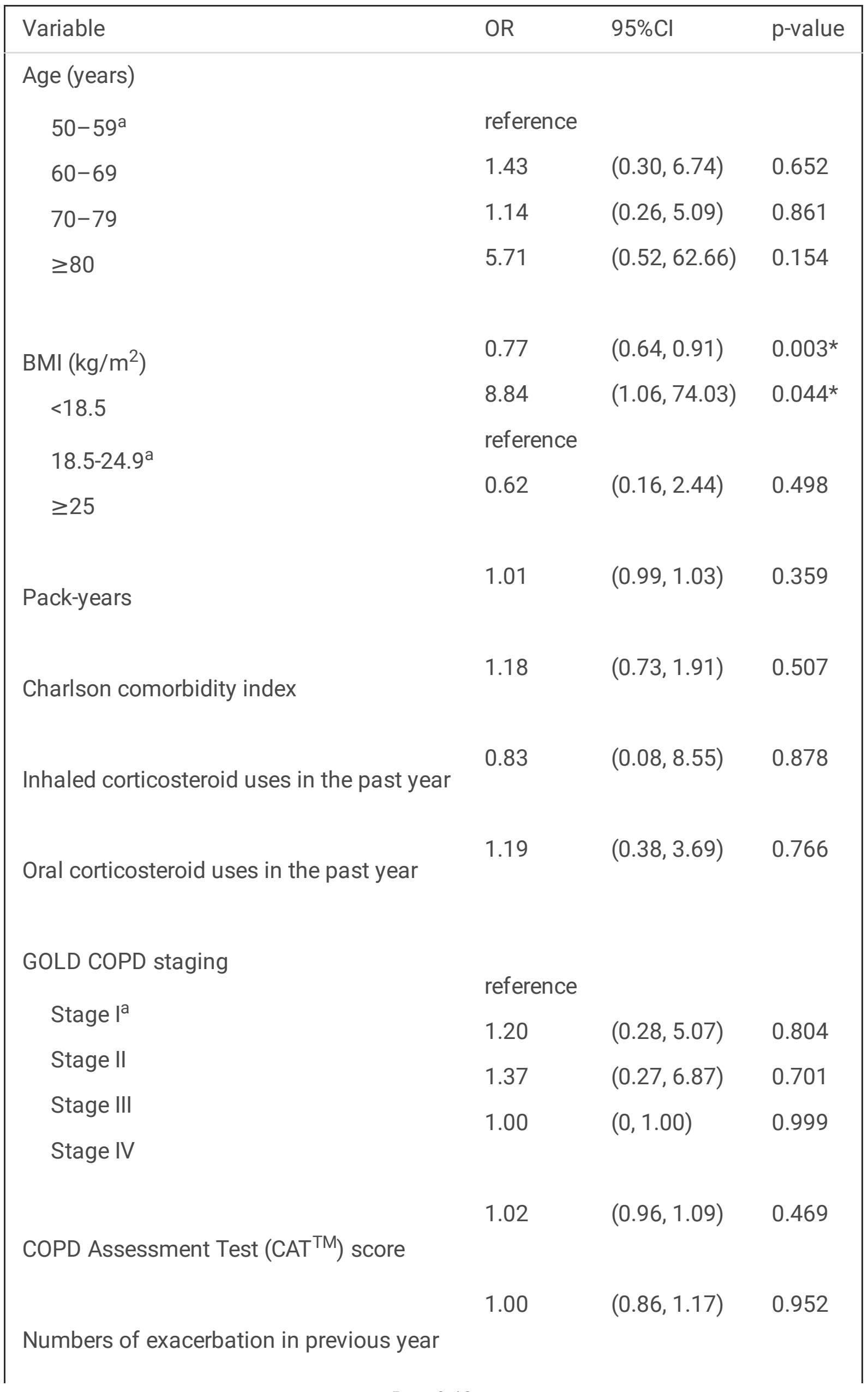




\begin{tabular}{|llll|} 
& 0.63 & $(0.44,0.89)$ & $0.009^{*}$ \\
FFMI $\left(\mathrm{kg} / \mathrm{m}^{2}\right)$ & 4.91 & $(1.26,19.06)$ & $0.022^{*}$ \\
$<17$ & reference & & \\
$\geq 17^{\mathrm{a}}$ & & & \\
\hline
\end{tabular}

a reference category

${ }^{*} \mathrm{p}$-value is significant.

$\mathrm{OR}=$ odd ratio, $\mathrm{Cl}=$ confidence interval

\section{Ability of calcaneus QUS to detect osteoporosis}

The mean T-score determined using calcaneus QUS was $-1.22 \pm 1.19$, which was significantly higher than the T-score determined using DXA at both the femoral neck and lumbar spine $(p<0.001)$ (Table 2$)$. The calcaneus QUS T-score was significantly correlated with the DXA T-score at the femoral neck $(r=0.62, p<$ $0.001)$ and lumbar spine $(r=0.53, p<0.001)$. Each QUS parameter was analyzed using ROC analysis to determine which QUS parameter was able to identify the participants with osteoporosis at the femoral neck, the lumbar spine, and either site (either the femoral neck or the lumbar spine). All four QUS parameters could discriminate osteoporosis at both sites, with an AUC ranging from 0.64 to 0.83 and $p<$ 0.05 , indicating statistical significance (see Figure 1). The AUC reflects the accuracy of the test. In general, an AUC closer to 1.0 indicates a more accurate test, while an AUC of 0.5 suggests the test has no discriminative ability. The accuracy of the QUS T-score was in an acceptable range to detect osteoporosis at either site (AUC 0.7-0.79).

Among all four QUS parameters, the T-score is commonly used in clinical practice by most physicians. In this study, we interpreted the result of the QUS T-score on the basis of the DXA T-score criteria. Using a DXA T-score $\leq-2.5$ as a reference for diagnosing osteoporosis, the prevalence of osteoporosis detected by calcaneus QUS was only $9 \%$, which was significantly lower than that detected by DXA at both the femoral neck and lumbar spine $(p<0.001)$. The sensitivity, specificity, positive predictive value, and negative predictive value of the different QUS T-score cutoff values are presented in Table 4. When a QUS T-score $\leq-2.5$ was used as the cutoff, the sensitivity was less than $15 \%$, while the specificity was approximately $95 \%$. The positive predictive value was $83.3 \%$, and the negative predictive value was approximately $29.5 \%-52.4 \%$. Therefore, subjects with a QUS T-score $\leq-2.5$ had a high likelihood of osteoporosis. However, with this cutoff level, calcaneus QUS missed diagnosing approximately $90 \%$ of the patients who were diagnosed with osteoporosis by DXA. When the QUS T-score cutoff value was increased, the sensitivity increased, while the specificity decreased. When the sensitivity exceeded $80 \%$, the specificity was less than $70 \%$ (Table 4 ).

Table 4 The ability of QUS T-score at the different cutoff to predict DXA-determined osteoporosis 


\begin{tabular}{|llllll|}
\hline Site & QUS & Sensitivity & Specificity & PPV & NPV \\
& T-score* & $(\%)$ & $(\%)$ & $(\%)$ & $(\%)$ \\
\hline Any site & -2.50 & 10.42 & 94.73 & 83.33 & 29.50 \\
& -1.25 & 70.83 & 73.68 & 87.18 & 50.00 \\
& -0.80 & 83.33 & 47.37 & 80.00 & 52.94 \\
& -0.09 & 91.66 & 31.57 & 77.19 & 60.00 \\
& & & & & \\
Femoral neck & -2.50 & 11.62 & 95.83 & 83.33 & 37.70 \\
& -1.25 & 72.09 & 66.67 & 79.49 & 57.14 \\
& -0.80 & 83.72 & 41.67 & 72.00 & 58.82 \\
& -0.09 & 93.02 & 29.17 & 70.18 & 70.00 \\
& & & & & \\
& -2.50 & 14.70 & 96.97 & 83.33 & 52.46 \\
& -1.25 & 85.29 & 69.70 & 74.36 & 82.14 \\
& -0.88 & 91.18 & 45.45 & 63.27 & 83.33 \\
& -0.09 & 97.06 & 27.27 & 57.89 & 90.00 \\
\hline
\end{tabular}

*The threshold value of QUS T-score below this point, osteoporosis is diagnosed by calcaneus QUS. PPV = positive predictive value, NPV = negative predictive value

\section{Discussion}

In the present study, the prevalence of osteoporosis determined by the gold standard DXA was $71.6 \%$, which was much higher than that previously reported for the Thai male population $(12 \%)^{15}$. The results from previous studies conducted in different countries using DXA showed that the prevalence of osteoporosis in COPD patients varied from $14-66 \%{ }^{5}$. The significant factors associated with osteoporosis in this study were $\mathrm{BMI}<18.5 \mathrm{~kg} / \mathrm{m}^{2}(\mathrm{OR}=8.84)$ and $\mathrm{FFMl}<17 \mathrm{~kg} / \mathrm{m}^{2}(\mathrm{OR}=0.63)$. A metaanalysis of two studies also demonstrated a similar result: having a $\mathrm{BMI}<18.5$ increased the risk of osteoporosis by 4.26 -fold $4,16,17$. FFMI (FFMI $<16 \mathrm{~kg} / \mathrm{m}^{2}$ ) was a risk factor associated with osteoporosis in COPD patients in two studies ${ }^{18,19}$. We found that FFMI $<17 \mathrm{~kg} / \mathrm{m}^{2}$, even when it was not defined as a depleted FFMI, increased the risk of osteoporosis in COPD patients. Regardless of the risk factors for osteoporosis, COPD patients have an approximately 2.8 times higher risk of osteoporosis than do those without COPD. Therefore, patients with COPD should be screened for osteoporosis ${ }^{4}$. 
According to the Thai Osteoporosis Foundation (TOPF), the BMD of males should be measured by DXA after the age of $70^{20}$. In this study, $52 \%$ of the male patients were older than 70 years. Another possible indication for a BMD assessment in COPD patients is prolonged glucocorticoid use (prednisolone 7.5 $\mathrm{mg} /$ day for at least three consecutive months). The patients in this study were prescribed glucocorticoids when they had an acute exacerbation, and they did not take the glucocorticoids for more than three months. Therefore, most COPD patients in this study had two main issues regarding DXA: (1) they had no indication for DXA measurements, and (2) DXA machines are scarce in rural areas and are usually available at tertiary care hospitals in large cities. As a result, COPD patients living in rural areas may have difficulty traveling to a city, especially if they live far from a city. Being old and experiencing breathlessness in COPD patients are also limitations to traveling long distances. The cost also increases when patients must pay for transportation. We believe that COPD patients in other countries also have similarly limited accessibility to DXA. Therefore, the calcaneus QUS was investigated in this study to determine (1) its ability to identify COPD patients with DXA-determined osteoporosis and (2) its suitability as a prescreening tool for osteoporosis in COPD patients.

There are four calcaneus QUS parameters (SOS, BUA, OSI, and T-score). The present study demonstrated that all the calcaneus QUS parameters can identify osteoporosis in COPD patients, with significant AUCs ranging from 0.646 (for OSI) to 0.835 (for SOS). An AUC greater than 0.7 reflects an acceptable level of accuracy of QUS. The AUCs of all the QUS parameters in this study determined using the AOS-100 device were comparable to those reported in previous studies using QUS devices from other manufacturers ${ }^{21,22}$. SOS had the highest AUC compared to the other QUS parameters in discriminating osteoporosis at any site and the femoral neck. The accuracy of the QUS T-score in identifying osteoporosis at the lumbar spine (AUC $=0.793$ ) was higher than that in identifying osteoporosis at the femoral neck (AUC $=0.706$ ) and either site (AUC $=0.723$. Previous study has also demonstrated the AUC of the QUS T-score in identifying osteoporosis at the lumbar spine is higher than that at the femoral neck ${ }^{23}$. We found a moderate correlation between the QUS T-score and DXA T-score at both the femoral neck and spine. The degree of correlation was higher for the femoral neck, which was consistent with the result in previous studies ${ }^{22,24}$.

In this study, we compared the DXA T-score with the QUS T-score because few studies have used the AOS100 device, and a previous study that used this device assessed the QUS T-score with respect to the DXA T-score ${ }^{25}$. When the DXA T-score cutoff value was used for diagnosis, in this study, the prevalence of osteoporosis and that of osteopenia determined using calcaneus QUS were $6 \%$ and $58.2 \%$, respectively, which were significantly lower than the prevalence determined using the gold standard DXA. The results from previous studies in COPD patients that used calcaneus QUS devices made by other manufacturers and interpreted the QUS T-score with respect to the DXA T-score showed that the prevalence of osteoporosis varied from 8.7-35\% and the prevalence of osteopenia varied from $35-51.35 \% 18,26,27$. However, previous studies did not compare the accuracy of QUS to that of DXA. The present study is the first to explore the utility of calcaneus QUS compared to that of DXA for detecting osteoporosis in COPD patients. 
We found that when a T-score $\leq-2.5$ was used as the cutoff for diagnosing osteoporosis, calcaneus QUS had an extremely low sensitivity for detecting DXA-defined osteoporosis. The negative predictive value was relatively low. As a result, most of the subjects classified as nonosteoporotic on the basis of the QUS T-score (QUS T-score > -2.5) additionally needed to undergo a DXA scan to confirm the absence of osteoporosis. Because the QUS T-score cutoff value differs by the device used, there is no single cutoff value that can be applied without errors in the number of COPD patients diagnosed with osteoporosis. Many studies also agree that the DXA T-score cutoff value cannot be used as the QUS T-score cutoff value $^{9,10}$. Different QUS devices yield different results. When a T-score threshold of $\leq-2.5$ was used for different QUS devices, the prevalence of osteoporosis varied by more than 10 -fold ${ }^{9}$. Different QUS devices also demonstrated different sensitivities, ranging from 34.8 to $88.4 \%$, and specificities, ranging from 41.2 to $91.9 \%$, when a QUS T-score cutoff of -2.5 was used ${ }^{10,28,29}$. The QUS measurement also varies by the skeletal site ${ }^{22}$. Therefore, calcaneal QUS cannot replace DXA for the diagnosis of osteoporosis according to the WHO classification.

Many studies have demonstrated that calcaneus QUS may be used as a prescreening tool before DXA $10,22,23$. With a device-specific upper threshold of $90 \%$ sensitivity and a lower threshold of $90 \%$ specificity, individuals who have either a high or low likelihood of osteoporosis can be identified ${ }^{9,30}$. However, this study has a relatively small sample size to determine an appropriate cutoff that achieves sufficient statistical power ${ }^{10}$. This study is also limited to male COPD patients in Thailand undergoing scans with the AOS-100 device. Due to the variety of QUS devices and cutoffs, calcaneus QUS prescreening algorithms must be based on a device-specific cutoff that has been validated in the populations for whom they are intended to be used ${ }^{10}$. Future studies in a larger cohort of COPD patients are needed to identify appropriate device-specific cutoffs for a prescreening algorithm.

\section{Conclusion}

This study demonstrated an ability of calcaneus QUS T-score to identify osteoporosis with acceptable level of accuracy. However, the sensitivity and specificity of QUS T-score were not sufficient high to serve as an alternative diagnostic tool to DXA. The ability of calcaneus QUS to serve as an alternative diagnostic tool to DXA is limited by the lack of consensus on which QUS device and diagnostic cutoff is best.

\section{Abbreviations}

$\mathrm{COPD}=$ Chronic obstructive pulmonary disease, $\mathrm{FEV} 1=$ Force expiratory volume in the first second, $\mathrm{FVC}=$ Force vital capacity, $\mathrm{BMI}=$ body mass index $\mathrm{BMD}=$ Bone mass density, FFMI = Fat free mass index, 25(OH)D = 25-hydroxyvitamin D, DXA = dual-energy X-ray Absorptiometry, QUS = Quantitative ultrasound, SOS = speed of sound, BUA = broadband ultrasound attenuation, OSI = osteo sono-assessment index

\section{Declarations}


Acknowledgments

This study was fully supported by grants from Walailak University (WU-IRG-62-007). The authors are grateful to the volunteers for participating in this study. The authors wish to thank the following persons from Thasala Hospital for their generous contributions to the study: the hospital director, the nurses at the COPD clinic, and the authorities at the rehabilitation center. The authors would also like to thank the hospital director, nurses, and nurse assistants at Walailak University Hospital.

\section{Author information}

\section{Affiliations}

\section{School of Medicine, Walailak University}

222, Thai Buri, Tha Sala, Nakhon Si Thammarat, 80160, Thailand

Wandee Chanprasertpinyo, Chuchard Punsawad, Naparat Sukkriang, Rapheeporn Khwanchuea, Pirada Yincharoen, Chaiwat Rerkswattavorn

\section{Walailak University Hospital}

222, Thai Buri, Tha Sala, Nakhon Si Thammarat, 80160, Thailand

Wandee Chanprasertpinyo, Naparat Sukkriang, Pirada Yincharoen, Chaiwat Rerkswattavorn

\section{Contributions}

W.C. contributed to study design, data collection, data interpretation, and writing of the manuscript. N.S. contributed to study design and data collection. C.P., P.Y., and R.K. contributed to the data collection. C.R. performed the data analysis and interpretation. All authors read and approved the final manuscript.

\section{Corresponding author}

Correspondence to Chaiwat Rerkswattavorn

\section{Ethics declarations}

\section{Competing interests}

All authors report no conflicts of interest related to this work.

\section{References}

1. Adeloye, D. et al. Global and regional estimates of COPD prevalence: Systematic review and metaanalysis. Journal of global health.5, 020415 https://doi.org/10.7189/jogh.05-020415 (2015). 
2. Singh, D. et al. Global Strategy for the Diagnosis, Management, and Prevention of Chronic Obstructive Lung Disease: the GOLD science committee report 2019. The European respiratory journal.53, https://doi.org/10.1183/13993003.00164-2019 (2019).

3. Okazaki, R., Watanabe, R. \& Inoue, D. Osteoporosis Associated with Chronic Obstructive Pulmonary Disease. J Bone Metab.23, 111-120 https://doi.org/10.11005/jbm.2016.23.3.111 (2016).

4. Chen, Y. W., Ramsook, A. H., Coxson, H. O., Bon, J. \& Reid, W. D. Prevalence and Risk Factors for Osteoporosis in Individuals With COPD: A Systematic Review and Meta-analysis. Chest.156, 10921110 https://doi.org/10.1016/j.chest.2019.06.036 (2019).

5. Bitar, A. N., Sulaiman, S., Ali, S. A., Khan, I. A. H., Khan, A. H. \& I. \& Osteoporosis among Patients with Chronic Obstructive Pulmonary Disease: Systematic Review and Meta-analysis of Prevalence, Severity, and Therapeutic Outcomes. J Pharm Bioallied Sci.11, 310-320 https://doi.org/10.4103/jpbs.JPBS_126_19 (2019).

6. Inoue, D., Watanabe, R. \& Okazaki, R. COPD and osteoporosis: links, risks, and treatment challenges. Int J Chron Obstruct Pulmon Dis.11, 637-648 https://doi.org/10.2147/COPD.S79638 (2016).

7. Sarkar, M., Bhardwaj, R., Madabhavi, I. \& Khatana, J. Osteoporosis in chronic obstructive pulmonary disease. Clin Med Insights Circ Respir Pulm Med.9, 5-21 https://doi.org/10.4137/CCRPM.S22803 (2015).

8. Chin, K. Y. \& Ima-Nirwana, S. Calcaneal quantitative ultrasound as a determinant of bone health status: what properties of bone does it reflect? Int J Med Sci.10, 1778-1783 https://doi.org/10.7150/ijms.6765 (2013).

9. Krieg, M. A. et al. Quantitative ultrasound in the management of osteoporosis: the 2007 ISCD Official Positions. Journal of clinical densitometry: the official journal of the International Society for Clinical Densitometry.11, 163-187 https://doi.org/10.1016/j.jocd.2007.12.011 (2008).

10. Thomsen, K. et al. Is calcaneal quantitative ultrasound useful as a prescreen stratification tool for osteoporosis? Osteoporosis international: a journal established as result of cooperation between the European Foundation for Osteoporosis and the National Osteoporosis Foundation of the USA 26, 1459-1475, doi:10.1007/s00198-014-3012-y (2015).

11. Charlson, M. E., Pompei, P., Ales, K. L. \& MacKenzie, C. R. A new method of classifying prognostic comorbidity in longitudinal studies: development and validation. Journal of chronic diseases.40, 373-383 https://doi.org/10.1016/0021-9681(87)90171-8 (1987).

12. Cummings, S. R., Bates, D. \& Black, D. M. Clinical Use of Bone DensitometryScientific Review. JAMA.288, 1889-1897 https://doi.org/10.1001/jama.288.15.1889 (2002).

13. Expert Panel on the, Identification, E., Overweight, T. \& Adults, O. i. Executive Summary of the Clinical Guidelines on the Identification, Evaluation, and Treatment of Overweight and Obesity in Adults. Archives of Internal Medicine.158, 1855-1867 https://doi.org/10.1001/archinte.158.17.1855 (1998).

14. Baarends, E. M., Schols, A. M., Mostert, R. \& Wouters, E. F. Peak exercise response in relation to tissue depletion in patients with chronic obstructive pulmonary disease. The European respiratory journal.10, 2807-2813 https://doi.org/10.1183/09031936.97.10122807 (1997). 
15. Pongchaiyakul, C. et al. Prevalence of osteoporosis in Thai men. Journal of the Medical Association of Thailand $=$ Chotmaihet thangphaet.89, 160-169 (2006).

16. Ogura-Tomomatsu, H. et al. Predictors of Osteoporosis and Vertebral Fractures in Patients Presenting with Moderate-to-Severe Chronic Obstructive Lung Disease. COPD: Journal of Chronic Obstructive Pulmonary Disease.9, 332-337 https://doi.org/10.3109/15412555.2012.667850 (2012).

17. Hattiholi, J. \& Gaude, G. S. Prevalence and correlates of osteoporosis in chronic obstructive pulmonary disease patients in India. Lung India.31, 221-227 https://doi.org/10.4103/09702113.135759 (2014).

18. Vrieze, A., de Greef, M. H. G., Wýkstra, P. J. \& Wempe, J. B. Low bone mineral density in COPD patients related to worse lung function, low weight and decreased fat-free mass. Osteoporos. Int.18, 11971202 https://doi.org/10.1007/s00198-007-0355-7 (2007).

19. Graat-Verboom, L. et al. Correlates of osteoporosis in chronic obstructive pulmonary disease: An underestimated systemic component. Respiratory medicine.103, 1143-1151 https://doi.org/10.1016/j.rmed.2009.02.014 (2009).

20. Songpatanasilp, T. et al. Thai Osteoporosis Foundation (TOPF) position statements on management of osteoporosis. Osteoporosis and Sarcopenia.2, 191-207 https://doi.org/10.1016/j.afos.2016.10.002 (2016).

21. Oral, A. et al. The ability of calcaneal and multisite quantitative ultrasound variables in the identification of osteoporosis in women and men. Turkish journal of physical medicine and rehabilitation.65, 203-215 https://doi.org/10.5606/tftrd.2019.1894 (2019).

22. Steiner, B., Dimai, H. P., Steiner, H., Cirar, S. \& Fahrleitner-Pammer, A. Prescreening for Osteoporosis With Quantitative Ultrasound in Postmenopausal White Women. Journal of ultrasound in medicine: official journal of the American Institute of Ultrasound in Medicine.38, 1553-1559 https://doi.org/10.1002/jum.14844 (2019).

23. Fitzgerald, G. E., Anachebe, T., McCarroll, K. G. \& O'Shea, F. Calcaneal quantitative ultrasound has a role in out ruling low bone mineral density in axial spondyloarthropathy. Clinical rheumatology.https://doi.org/10.1007/s10067-019-04876-9 (2020).

24. Cetin, A., Ertürk, H., Celiker, R., Sivri, A. \& Hasçelik, Z. The role of quantitative ultrasound in predicting osteoporosis defined by dual X-ray absorptiometry. Rheumatology international.20, 55-59 https://doi.org/10.1007/pl00006857 (2001).

25. Chang, H. C. et al. Does coffee drinking have beneficial effects on bone health of Taiwanese adults? A longitudinal study. BMC Public Health.18, 1273 https://doi.org/10.1186/s12889-018-6168-0 (2018).

26. Ramachandran, K., Mani, S. K., Gopal, G. K. \& Rangasami, S. Prevalence of Bone Mineral Density Abnormalities and Factors Affecting Bone Density in Patients with Chronic Obstructive Pulmonary Disease in a Tertiary Care Hospital in Southern India. J Clin Diagn Res.10, 0C32-0C34 https://doi.org/10.7860/JCDR/2016/22464.8551 (2016). 
27. Bhattacharyya, P. et al. Prevalence of osteoporosis and osteopenia in advanced chronic obstructive pulmonary disease patients. Lung India.28, 184-186 https://doi.org/10.4103/0970-2113.83974 (2011).

28. Díez-Pérez, A. et al. Evaluation of calcaneal quantitative ultrasound in a primary care setting as a screening tool for osteoporosis in postmenopausal women. Journal of clinical densitometry: the official journal of the International Society for Clinical Densitometry.6, 237-245 https://doi.org/10.1385/jcd:6:3 (2003).

29. Felder, M., Haldemann, R. \& Anderhub, H. P. [Value of ultrasound study and dual energy x-ray absorptiometry (DEXA) for assessment of risk of osteoporosis]. Praxis.89, 233-239 (2000).

30. Clowes, J. A., Peel, N. F. \& Eastell, R. Device-specific thresholds to diagnose osteoporosis at the proximal femur: an approach to interpreting peripheral bone measurements in clinical practice. Osteoporosis international: a journal established as result of cooperation between the European Foundation for Osteoporosis and the National Osteoporosis Foundation of the USA.17, 1293-1302 https://doi.org/10.1007/s00198-006-0122-1 (2006).

\section{Figures}




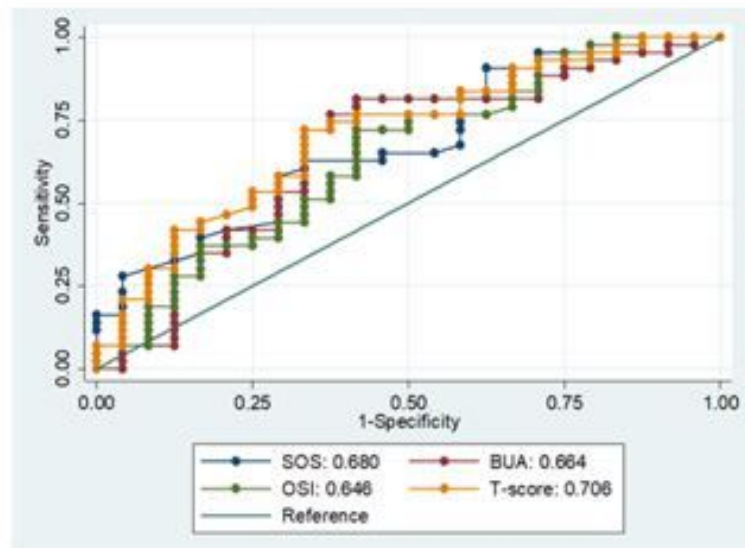

(a) Femoral neck

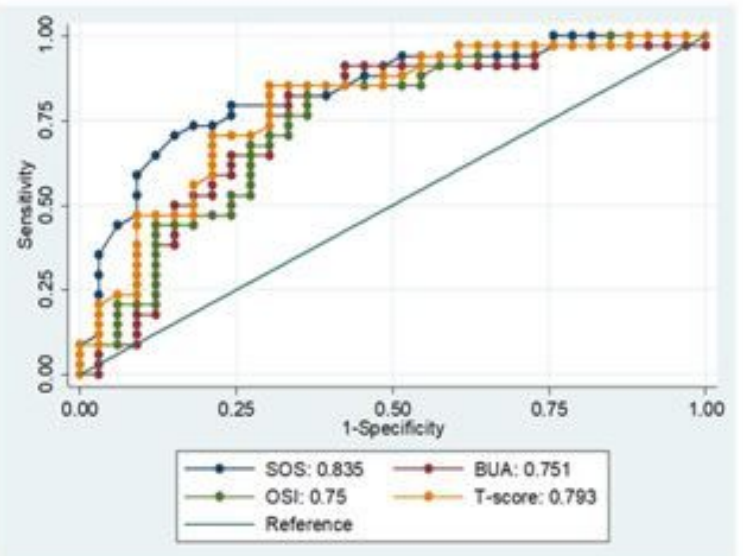

(b) Lumbar spine

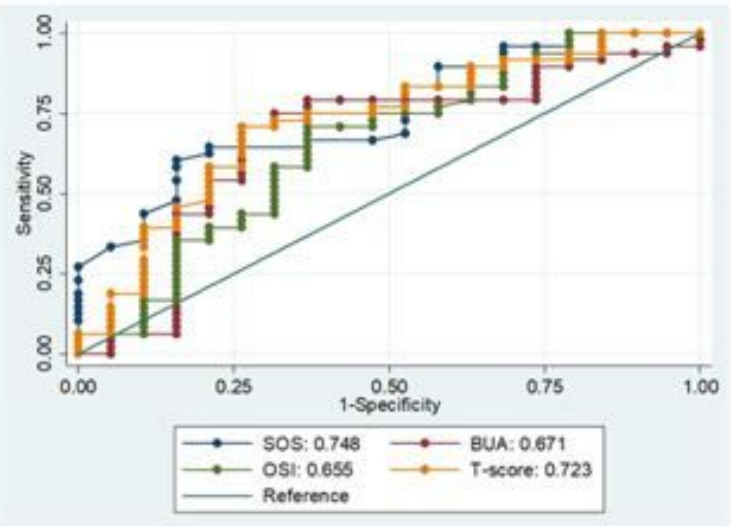

(C) Any site

\section{Figure 1}

Receiver operating characteristics (ROC) curves for each QUS parameter (BUA, SOS, OSI, T-score) for detecting osteoporosis at (a) Femoral neck, (b) lumbar spine, and (c) Any site (defined as T-score $\leq-2.5$ at either femoral neck or lumbar spine) 